\title{
Frontières
}

\section{Vers une nécropoétique}

\section{Le cadavre comme "figure de vérité »?}

\section{Isabelle-Rachel Casta}

Volume 23, numéro 2, printemps 2011

Enquêtes sur le cadavre : 2. Fantastique

URI : https://id.erudit.org/iderudit/1007592ar

DOI : https://doi.org/10.7202/1007592ar

Aller au sommaire du numéro

Éditeur(s)

Université du Québec à Montréal

ISSN

1916-0976 (numérique)

Découvrir la revue

Citer cet article

Casta, I.-R. (2011). Vers une nécropoétique : le cadavre comme " figure de vérité » ? Frontières, 23(2), 59-63. https://doi.org/10.7202/1007592ar d'utilisation que vous pouvez consulter en ligne.

https://apropos.erudit.org/fr/usagers/politique-dutilisation/ 


\section{VERS UNE NÉCROPOÉTIQUE Le cadavre comme «figure de vérité »?}

\author{
Isabelle-Rachel Casta, Ph.D., \\ professeure à l'Université d'Artois, Laboratoire Texte et Culture.
}

Les séries télévisées et les thrillers contemporains font la part belle au corps mort, au corps du mort, amenant la critique à s'interroger sur cette «surcadavérisation ». Faut-il y voir un avatar de notre hantise traditionnelle pour les morts sans sépulture, errants et zombies de toute nature? N'y a-t-il pas plutôt, conjointement à la laïcisation rapide de nos sociétés, un appel diffus à la connaissance, ésotérique ou eschatologique, qui remplacerait l'ancienne foi de notre candeur passée? Quoi qu'il en soit, philosophes et thanatologues érigent bien le corps en "figure de vérité", et la morgue devient le lieu romanesque incontournable où se déroulent les fastes inquiétants de la cérémonie médico-légale. Le légiste en rédempteur campe une nouvelle figure héroïque, à la fois toute-puissante par son savoir technique, mais démunie et vulnérable devant le mystère impénétrable de nos fins dernières.

Comme toujours après une autopsie, on était silencieux

et on évitait de se regarder dans les yeux. [...] Un cadavre

est un message clandestin. Passé en fraude à la frontière de

la mort, le corps raconte l'histoire de l'être humain

qui vivait il y a si peu de temps (Hettche, 2003, p. 26).

La puissante ambiguiité qui gouverne notre confrontation au corps mort, au corps du mort, revêt à mon sens deux figures littéraires particulièrement riches en enseignement: la fascination épouvantée pour l'objet-cadavre (et son cortège de fictions exorcisantes sur les spectres, zombies, vampires, revenants... qui n'en sont que la textualisation fantasmagorique), puis la transformation de la répulsion en gratitude, lorsque le cadavre hideux redevient une figure herméneutique majeure, lourd d'une vérité que nous accueillons enfin sans réticence.

\section{CORPS SANS SÉPULTURE...}

Les grandes figures littéraires de médecins dévoués ou démoniaques touchent désormais aux mythèmes (Balzac, Zola, Wells, A.J. Cronin...), le versant ensoleillé de l'isotopie se noyant peu à peu sous les ombres inquiétantes de Caligari ${ }^{1}$ ou de $\mathrm{Mabuse}^{2}$, nécromants pseudo-scientifiques au fort parfum de thaumaturges. Or, depuis quelques années (on songera au Necropolis de Herbert Liebermann [1977] comme origine acceptable) domine la figure du légiste, ami de la décomposition, de la puanteur et des mouches. Faut-il voir dans cette émergence l'indice d'une mutation irréversible de notre sensibilité ? La laïcisation de nos sociétés nous laisse-t-elle à ce point démunis devant la «charogne » que nous reportons sur le médecin des morts le tremendum et le fascinans, jadis réservés aux prêtres?

La surabondance des séries à succès qui se déroulent, en tout ou en partie, dans une morgue et/ou une entreprise d'embaumement nous adresse en tout cas un signe fort: l'I.M.L. ${ }^{3}$ est le «it» lieu, le cadre fictionnel qui fait rêver et frissonner. Songeons qu'aujourd'hui nous sommes, socialement et anthropologiquement, très peu en présence des morts, moins encore au «contact » de ces derniers; on ne meurt plus chez soi, les veillées mortuaires s'estompent, et la surmédicalisation de nos fins dernières retranche le mourant de la communauté des hommes: cachez cette agonie, que je ne saurais voir! Ce qui amène peut-être une sorte de compulsion de réparation, exercée par le truchement de professionnels hypercompétents, cette Tru Davis ${ }^{4}$, ce Letzte $Z_{\text {Zuge }}^{5}$, cette Temperance Brennan ${ }^{6}$ surdouée, ou cette famille Fisher ${ }^{7}$ tout entière qui dévale joyeusement les escaliers le matin pour aller embaumer les morts qui reposent au sous-sol... Qu'ils soient légistes ou thanatopracteurs, tous ont en commun d'avoir dépassé, depuis longtemps, le stade banal de l'effroi, du dégoût, de l'ignorance crasse. 
Il n'a de toute façon jamais été simple de surmonter l'interdit qui frappe le corps du mort pour procéder à son autopsie; l'historien Grégoire Chamayou (2008) nous le rappelle crûment, en évoquant, à propos de l'expérimentation en général, la devise de certains légistes : facere experimentum in corpore vili, c'est-àdire : faisons des expériences sur un corps vil... On ajoutera : car seuls ceux-ci sont d'un accès aisé! Souvenons-nous de L'Impasse aux violences, film britannique de John Gilling (1959), où des brutes sadiques fournissent en cadavres «frais» un professeur de médecine sans scrupules... Cette "gène», souvent transformée en exploitation sociale mal déguisée (mendiants, filles de joie, déments ou condamnés fournissant les gros bataillons des fameux «corps vils»), se traduit aujourd'hui par une proclamation sans cesse réaffirmée du respect dû aux morts, de l'intimité spéciale entre le légiste et «son» cadavre, de la considération éprouvée et soulignée à tout instant entre le corps du mort et celui/celle qui va en violer l'enveloppe charnelle; le légiste va parfois jusqu'à s'imaginer à la place du mort: "L'idée vertigineuse la traversa soudain que c'était elle qu'elle voyait sur cette table. Qu'elle assistait à l'autopsie de son propre cadavre » (Gerritsen, 2007, p. 53).

En contrepartie, les contraintes et servitudes du métier sont hautement manifestes, comme s'il fallait payer cher le privilège particulier qui consiste à manier la scie Stryker et le kit de thoracotomie dans le fracas de la soufflerie et des outils métalliques, sous la lumière crue des lampes: «Depuis sept heures du matin elle respirait l'arôme de la mort, tellement familier qu'elle ne broncha pas lorsque se lame incisa la peau froide et qu'une odeur nauséabonde s'échappa des organes mis à nu » (Gerritsen, 2009, p. 16). Autres exemples (on pourrait les multiplier quasi à l'infini, la scène d'autopsie faisant florès dans la littérature contemporaine): «De la matière cérébrale collait aux manches de la blouse maculée de sang que portait le Dr Kay Scarpetta [...] à l'instant où elle l'avait incisée, elle avait perçu cette odeur à la fois âcre et écœurante de l'alcool en cours de métabolisation» (Cornwell, 2009, p. 11); "Le rectum est fortement élargi, saignant dans la partie inférieure, et la partie supérieure est occupée par une matière fécale ayant l'aspect d'une bouillie verdâtre. Une odeur âcre envahissait à présent la pièce [...]» (Hettche, 2003, p. 24).

On le voit, l'ekphrasis de l'autopsie suit à peu près toujours le même schéma : dégoût du profane, impassibilité du légiste, déploration de la vénusté détruite de la victime, précision hyperréaliste des gestes et des instruments (c'est l'effet Urgences, ses " gaz du sang» et ses «O Neg»), odeurs épouvantables-il y aurait de quoi rédiger une anthologie - et pour finir résultats du labo et ultima verba sur la condition humaine, fragile ${ }^{8}$, et les pouvoirs du légiste, immenses mais dérisoires.

Notre hantise de la chair putréfiée, où la rigidité du cadavre est «animée » des nuées d'insectes et des parasites qui grouillent dans tous les orifices - un «ver de cercueil » peut sauter 90 fois sa taille-se thématise particulièrement dans les films de zombies, qui précisément répondent au patron stylistique de l'adynaton, la figuration de l'impossible: ils marchent, agissent, tout en se défaisant sous nos yeux! Ils conjuguent donc deux horreurs: celle du corps sans sépulture qui promène parmi les vivants

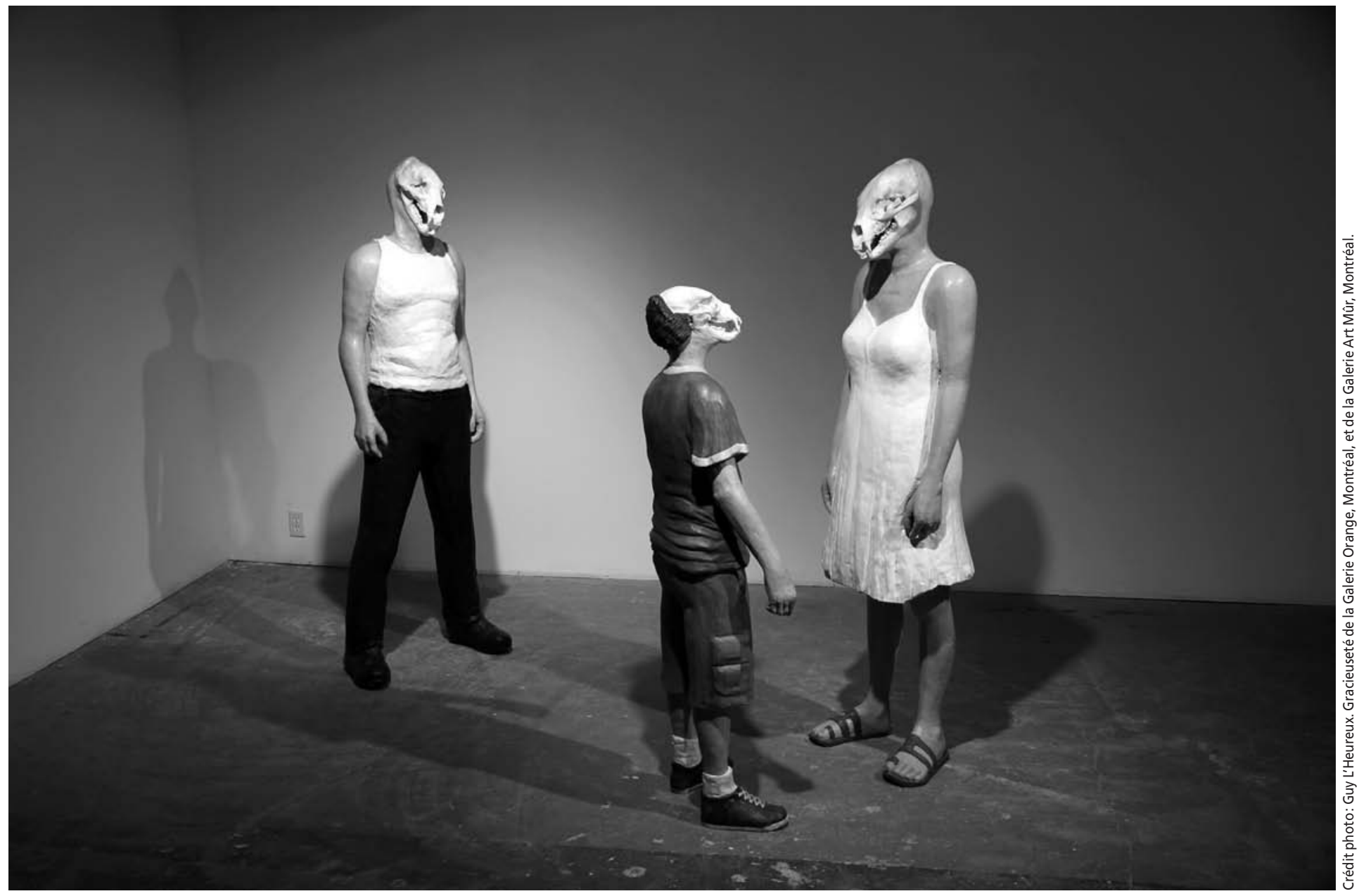

Jean-Robert Drouillard, Le Père, 2010, acrylique sur tilleul et résine d'époxy, crâne d'ours noir, 169 x 46 × 30 cm; La Mère et l'Enfant, 2010, acrylique sur tilleul et résine d'époxy, crâne d'ours noir, 163 × 36 × 27 cm, 132 × 41 x $19 \mathrm{~cm}$. 
QUE L'INFINIMENT PETIT, LE NÉGLIGEABLE,

LE QUASI INVISIBLE DÉLIVRE LE SECRET

DE NOTRE FIN EST L'UN DES PARADOXES

DE L'INVESTIGATION POST MORTEM.

le travail de la décomposition et celle de la férocité supposée des morts envers leurs anciens "proches ", dont ils ne sont plus que les caricatures méconnaissables et corrodés. Plusieurs œuvres, récentes, ou plus anciennes, viennent appuyer nos dires: Diary of the Dead (George A. Romero, É.-U., 2008), Shaun of the Dead (Edgar Wright, R.-U./France, 2005), Land of the Dead (George A. Romero, France/É.-U./Canada, 2005), Dawn of the Dead (George A. Romero, Italie/É.-U., 1978)... sans remonter à la paradigmatique Nuit des morts-vivants (1968), qui a lancé Romero et terrifié les deux tiers de la planète! Cadavres au désespoir, cannibales et sans affects, ils représentent notre part nocturne, celle d'une apocalypse froide et nauséabonde. Le critique Paul Ardenne, en décentrant un petit peu l'objet de notre analyse, commente ainsi le (dé)goût du public pour les histoires de zombies: «Le revenant, c'est la représentation même de la vie mais après refonte, a posteriori, au terme d'un examen rétrospectif de la vie vécue. Une représentation dévoyée, dont la fonction est de rendre la vie à nouveau présente, sous condition d'une correction radicale» (Ardenne, 2006, p. 404).

C'est sans doute dans ce type de fantastique que se noue et se joue l'essence de notre rapport au corps du mort, privés que nous sommes de la transcendance de jadis, mais toujours aussi apeurés et démunis devant «ce qui n'a plus de nom dans aucune langue ». Comment, alors, transformer notre dépouille, pitoyable et méphitique, en interlocuteur digne de foi, en acteur de vérité et de cohérence?

\section{... EN FIGURE DE VÉRITÉ}

Ils ignoraient quasiment tout de l'homme étendu entre eux.

Ils ne savaient ni son nom, ni son histoire [...]

Et cependant, après l'autopsie, ils le connaîtraient

plus intimement que personne ne l'avait jamais connu.

(Gerritsen, 2009, p. 100).

Un constat s'impose sans difficulté: à travers films ${ }^{9}$, romans à foison et séries innombrables, le cadavre est devenu en quelques années un must dans le casting des œuvres à succès. La vague de fond a été préparée par les séries «médicales », types Docteur House ou Grey's Anatomy - et je citais plus haut la vétérane, Urgences, dont les 15 saisons se sont achevées récemment -, puis l'attention s'est déportée du malade vers le mort, et par voie de conséquence de l'urgentiste vers le coroner ou le thanatopracteur. La fascination récente ressentie pour le métier de légiste surprend un peu les praticiens: eux n'ont pas l'impression d'exercer une profession aussi attrayante, et ils s'en expliquent d'ailleurs dans Les Enquêteurs de l'ombre, un excellent documentaire d'Eléonore Manéglier (France, 2009). Pourtant l'image de Scully chaussant ses lunettes anti-projection dans $X$-Files continue de passionner les aficionados, tandis que les multiples descriptions de l'habillage, puis du déshabillage, des légistes chez P. Cornwell et K. Reichs confèrent à la pratique la pompe sacerdotale des instants sacrés ou des «mystères » gréco-latins.

L'entomologie forensique (nous adoptons cet anglicisme, en usage dans tous les compendia sur la question) pose la question de la vérité aux plus infimes créatures: les larves, leurs exuvies, leurs carapaces; ces asticots qui s'emparent de nous, de nos organes et de nos tissus mous dès que la mort nous a saisis sont

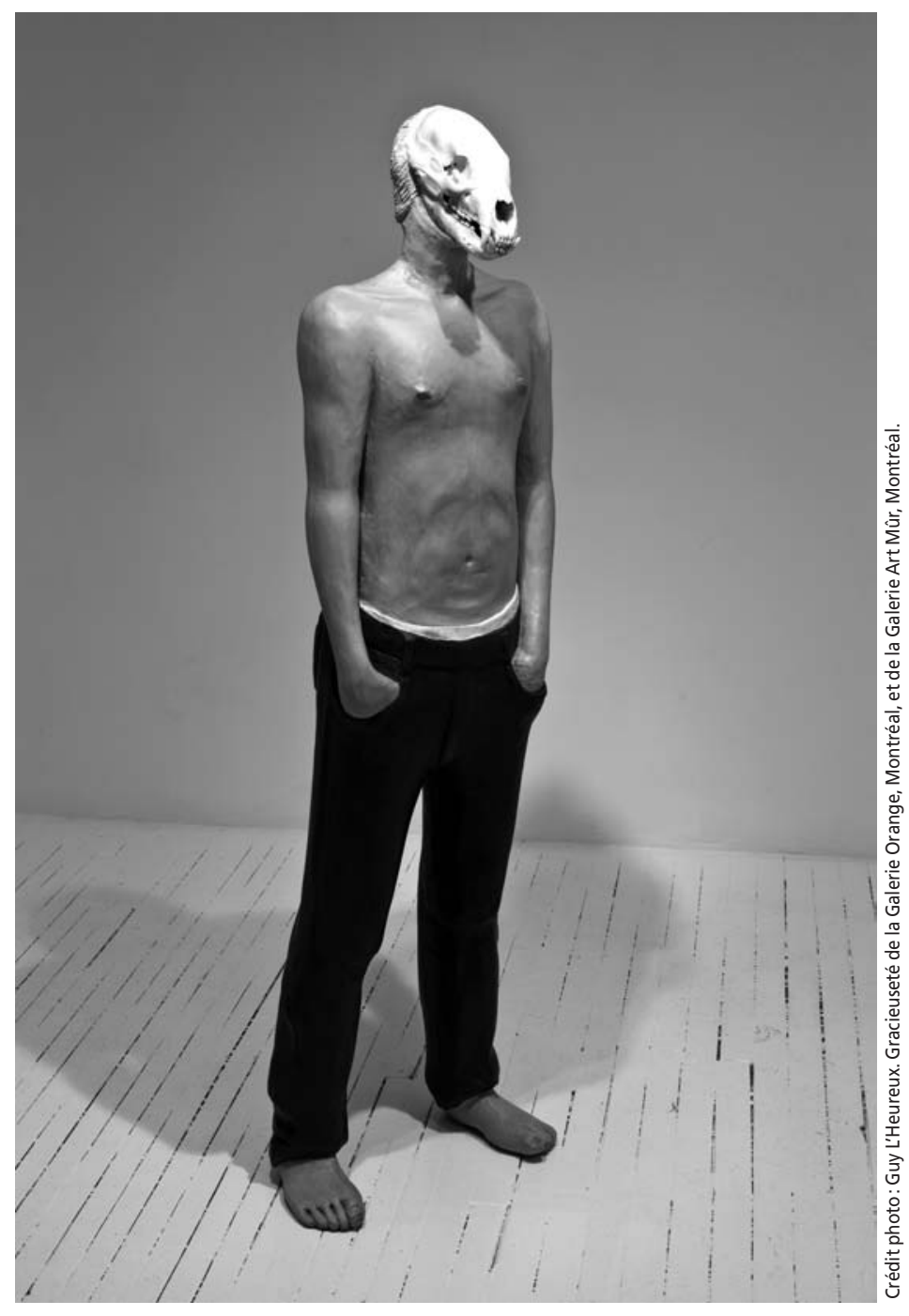

Jean-Robert Drouillard, L'Adolescent, 2010, acrylique sur tilleul et résine d'époxy, crâne d'ours noir, $155 \times 38 \times 27$ cm.

en effet les meilleurs alliés de la justice: ils diront quand a eu lieu le décès, où, dans quelles circonstances météorologiques... Ce sont les huit fameuses "escouades de la mort», mises en évidence par un vétérinaire, Jean-Pierre Mégnin, qui en deux ouvrages a jeté toutes les bases de l'entomologie légale (Faune des tombeaux, 1887, et La faune des cadavres: application de l'entomologie à la médecine légale, 1894). Que l'infiniment petit, le négligeable, le quasi invisible délivre le secret de notre fin est l'un des paradoxes de l'investigation post mortem. C'est sans doute l'une des raisons qui a motivé William Bass, l'anthropologue le plus célèbre d'Amérique: sa «ferme des corps ${ }^{10}$ » (Body Farm) est un lieu unique au monde, entièrement dévolu à la mise en situation de cadavres altérés, dans toutes les positions et tous les environnements possibles. Les visiteurs (triés sur le volet) marchent littéralement sur les corps, ce qui a amené Bass à accrocher une pancarte au message succinct: «NO CROSSCONTAMINATION!!!!!!!!» Comprendre: ne vomissez pas sur mes cadavres! vous mêleriez vos bactéries aux leurs...

Dans un autre genre, la fascination pour le cadavre maquillé, ou mis en scène, est surexploitée par le cinéma, qui y voit l'occasion de scènes gore ou trash promptes à flatter le voyeurisme complaisant du public: rappelons Anamorph (H. Miller, É.-U., 2007), thriller horrifique où les corps des victimes sont réarrangés comme dans un tableau de Francis Bacon-sans même parler de Seven, le chef d'œuvre ambigu et terrible de David Fincher. 
Il est permis de lire, dans ces outrages, la recherche dévoyée et pathologique d'une ultime vérité : la nécrophilie n'est pas loin, elle qui prétend exprimer encore une autre part de nos abîmes-c'est chose faite dans Coup de blues (P.T. Jefferson, 2000), où un prédateur sexuel embaume ses victimes avant de les violer.

Ces «accidents» (au sens philosophique) de la quête de vérité, qu'elle soit banalement policière ou plus métaphysique, réhabilitent en tout cas pleinement le corps du mort, ne l'externalisent plus du récit, comme un protagoniste gênant qui ne ferait qu'encombrer. Il est et reste le centre du processus, le maître muet et douloureux d'une lente catabase, le glyphe à décrypter par de patientes et savantes analyses. Ce n'est pas l'illusion ressuscitante des histoires de détective à la Leroux, ou des sagas vampiriques (Stephenie Meyer), mais la parole redonnée à celui qui n'a plus de voix, la lumen opacatum d'un départ définitif mais pour un instant différé: "Seul, si froid, environné de silence, l'homme mort attend, il m'attend. Je suis le dernier être auquel il se confiera avec des mots qui ne sont pas de ce monde [...] à la morgue où je déploierais tout mon talent pour le faire parler, à moi seule » (Cornwell, 2001, p. 115). Cela n'empêche pas David Le Breton d'insister sur la nécessaire froideur à maintenir entre légiste et autopsié, froideur qui rejoint l'anthropologique séparation qui règle depuis toujours le séjour des morts et celui des vivants: «L'homme disséqué est dépersonnalisé et perçu comme une matière inerte dont il faut déconstruire les éléments, démembrer la forme, il est un bel outil d'apprentissage. L'émotion est tenue à distance» (Le Breton, 1993). On trouve un écho de cette impersonnalisation forcée dans de nombreux romans, mais c'est généralement pour la réfuter peu après en affirmant que même le plus endurci des légistes est submergé par l'émotion, etc. : «La mort est donc pour moi chose habituelle. Son odeur, sa vue, son idée même me sont familières. Sur le plan des émotions, mon métier m'a endurcie. J'ai appris à rester détachée. Pourtant, quelque chose chez cette vieille femme faisait voler en éclat ma carapace» (Reichs, 2004, p. 13).

Faire allégeance à ceux qui sont partis «de l'autre côté» permet ensuite à toute l'infrastructure criminalistique de se déployer, dans ses fastes glaçants. Moins consensuel, Michel Houellebecq élève, lui, une sorte de "cantique de la pourriture» qui profère notre immanence d'être périssable, sans pour autant abdiquer notre grandeur: «Le monde pue. Il n'y a pas de fantôme sous la lune tumescente. Il n'y a que des cadavres gonflés, ballonnés et noirs, sur le point d'éclater dans un vomissement pestilentiel» (Houellebecq, 1999, p. 73).

Invasive et réparatrice, la nécropsie semble obéir à la fameuse dialectique hégelienne, la Aufhebung; il s'agit d'ôter d'abord quelque chose (ici, la forme humaine, l'intégrité du corps, le respect de son intangibilité, etc.) pour le restaurer ensuite plus complètement, plus profondément: le corps recousu est désormais pacifié, il a livré son ultime vérité, son secret, parfois le modus operandi de sa mise à mort, la signature de l'assassin; il est «subsumé» par rapport à l'état antérieur à l'autopsie: le même, mais changé en mieux, après un "passage dans la nuit du Sens », cette apparente altération de son éthos primitif. Il rejoint ainsi l'analyse du philosophe Michel Foucault, pour qui : «[... dans la hardiesse du geste qui ne viole que pour mettre à jour, le cadavre devient le plus clair moment dans les figures de la vérité » (Foucault, 1963, p. 126). La morgue est bien le lieu paradoxal de notre plus grande technicité, et de notre plus extrême vulnérabilité : l'homme y est roi, et mendiant.

\section{Bibliographie}

ARDENNE, P. (2001). L'Image corps, figures de l'humain dans l'art du vingtième siècle, Paris, éditions du Regard.

ARDENNE, P. (2006). Extrême, esthétique de la limite dépassée, Paris, Flammarion.

ARIÈS, P. (1977). L'Homme devant la mort, Paris, Seuil.

CASTA, I. (2007). Nouvelles Mythologies de la Mort, Paris, Champion.

CHAMAYOU, G. (2008). Les corps vils. Expérimenter sur les êtres humains aux XVII e et XIX $X^{e}$ siècles, Paris, La Découverte-Les empêcheurs de penser en rond.

COMAR, V. (1993). Les Images du corps, Paris, Gallimard.

CORNWELL, P. (1993). Mémoires mortes, trad. D. DUPONt-VIAU, Paris, éditions du Masque.

CORNWELL, P. (1995). La séquence des corps, trad. G. BERTON, Paris, Librairie des Champs-Élysées.

CORNWELL, P. (1998). Mordoc, trad. H. NARBONNE, Paris, Calmann-Lévy.

CORNWELL, P. (2001). Dossier Benton, trad. H. NARBONNE, Paris, Calmann-Lévy.

CORNWELL, P. (2004). Postmortem, trad. Andréa H. JAPP, Paris, éditions des Deux Terres.

CORNWELL, P. (2008). Registre des morts, trad. Andréa H. JAPP, Paris, éditions des Deux Terres.

CORNWELL, P. (2009). Scarpetta, trad. Andréa H. JAPP, Paris, éditions des Deux Terres.

EDELMAN, B. (2009). Ni chose ni personne. Le corps humain en question, Paris, Herman.

FOUCAULT, M. (1963). Naissance de la clinique: Une archéologie du regard médical, Paris, Presses universitaires de France.

GERRITSEN, T. (2007). Le lien fatal, trad. J. MARTINACHE, Paris, Presses de la Cité.

GERRITSEN, T. (2009). Au bout de la nuit, trad. H. TÉZENAS, Paris, Presses de la Cité.

GUIOMAR, M. (1988). Principes d'une esthétique de la mort, Paris, Corti.

HETTCHE, T. (2003). Le Cas Arbogast, trad. N. CASANOVA, Paris, Grasset.

HOUELLEBECQ, M. (1999). H.P. Lovecraft, Contre le monde, contre la vie, Paris, J'ai lu.

LE BRETON, D. (1993). La chair à vif, usages médicaux et mondains du corps humain, Paris, éditions A.M. Métailié.

LE BRETON, D. (2003). Anthropologie du corps et modernité, Paris, Presses universitaires de France.

MARC, B. (2009). Profession médecin légiste, Paris, Éditions Démos. MCKINNON, A. (2009). L'Enfant sans nom, trad. C. CHICHEREAU, Paris, Fleuve noir.

REICHS, K. (2002). Mortelles décisions, trad. V. MIKHALKOv, Paris, Robert Laffont.

REICHS, K. (2004). Secrets d'outre-tombe, trad. V. MIKHALKOv, Paris, Robert Laffont.

REICHS, K. (2009). Meurtres en Acadie, trad. V. MIKHALKOV, Paris, Robert Laffont.

ROACH, M. (2005). Macchabées, la vie mystérieuse des cadavres, trad. C. DENIARD, Paris, Calmann-Lévy. 


\section{Notes}

1. Le Cabinet du Docteur Caligari est un film expressionniste allemand de Robert Wiene (1919).

2. Personnage créé par le romancier Norbert Jacques, le Docteur Mabuse est surtout connu par les adaptations cinématographiques réalisées par Fritz Lang en 1922, 1933 et 1960.

3. Institut médico-légal; le terme de «morgue», plus sinistre et plus imagé, renvoie en fait au guichet des prisons d'où les gardiens «morguaient» (regardaient attentivement) les prisonniers...

4. Héroïne de la populaire série Tru Calling (devenue en français «Compte à rebours ", Jon Harmon Feldman, 2003); dans cette histoire semifantastique, une jeune assistante médico-légale, Tru Davis, entend les morts l'appeler au secours lorsqu'on les apporte à la morgue. Elle doit alors remonter le temps pour les empêcher de mourir, et se réveille subitement le matin même du jour déjà vécu; elle a donc 24 heures devant elle pour faire pièce au désastre...

5. Cette excellente série allemande (Stephan Bernhard, 1998) s'intitule en français Le dernier témoin ; il s'agit évidemment du légiste, seul autorisé à se pencher sur le corps ouvert et à scruter les organes, les fluides, les tissus... qui racontent l'histoire d'un être dans sa plus totale intimité, celle du physiologique.

6. Rendue mondialement célèbre par la série Bones (Hart Hanson, 2005), Temperance Brennan est anthropologue judiciaire dans les romans quasi autobiographiques de sa créatrice, Kathy Reichs. Rivale officielle de la Kay Scarpetta de Patricia Cornwell, Tempe est capable de reconstituer un corps entier à partir d'une molaire ou d'une vertèbre, et passe aisément de l'ontogenèse à la phylogenèse - ce que ne font pas forcément ses consœurs «seulement» légistes.
7. La série Six Feet Under (Six pieds sous terre, Alan Ball, 2001) a séduit et parfois choqué par la crudité de ses scènes sexuelles autant que par l'omniprésence des morts dans l'intimité de cette famille d'embaumeurs. Le générique inquiétant, les prologues inéluctablement meurtriers et l'atmosphère curieusement "provinciale» de l'ensemble tranchent radicalement avec les séries voisines, de type Preuves à l'appui ou Coroner da Vinci.

8. La méditation sur le cadavre écorché en salle d'autopsie ressemble aux anciennes vanités: "Comme l'os humain est vulnérable! pensa-t-elle. [...] l'une après l'autre, les côtes cèdent sous l'acier trempé. Nous sommes faits d'une matière fragile»(Gerritsen, 2007, p. 48).

9. Un exemple parmi cent: le film japonais Departures (Okuribito) a valu l'Oscar du meilleur film étranger à son réalisateur Yojiro Takita en racontant l'initiation à la thanatopraxie d'un jeune violoncelliste devenu croque-mort, maladroit et impressionnable...

10. Deux hectares près de Knoxville dans le Tennessee sont entièrement voués aux morts en figure de vérité; le nom technique de l'endroit est: le Forensic Anthropology Center of Tennessee, et il doit en grande partie sa célébrité au roman de $\mathrm{P}$. Cornwell qui lui est consacré: La séquence des corps (1995).

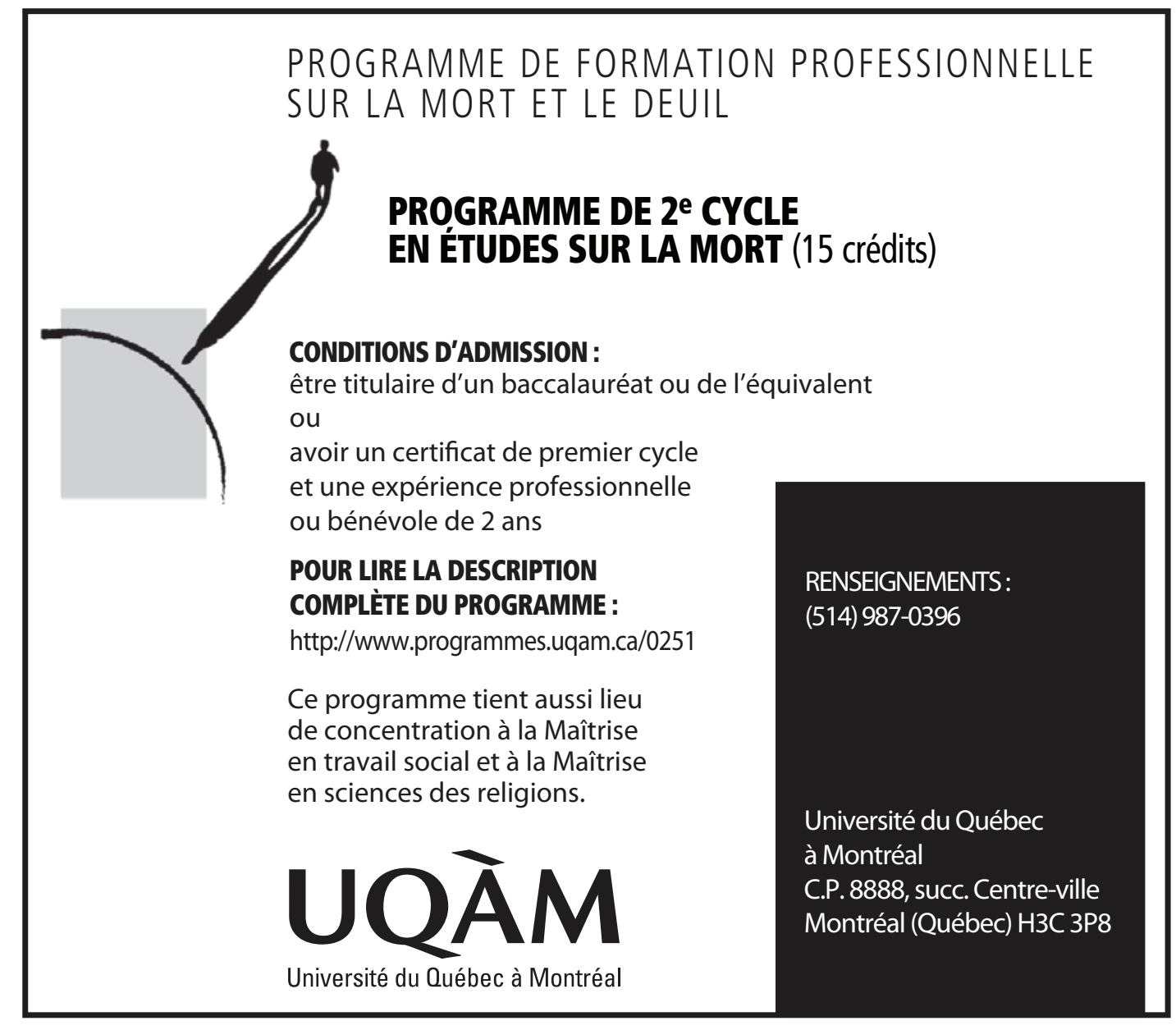

